\title{
A novel monoclonal antibody targeting a novel epitope of VE-cadherin inhibits vasculogenic mimicry of lung cancer cells
}

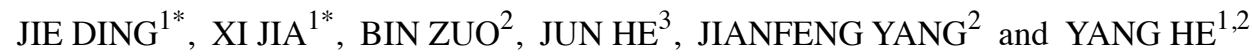 \\ ${ }^{1}$ Ministry of Health Key Laboratory of Thrombosis and Hemostasis, Jiangsu Institute of Hematology, \\ The First Affiliated Hospital of Soochow University, Suzhou, Jiangsu 215006; \\ ${ }^{2}$ Cyrus Tang Hematology Center and Ministry of Education Engineering Center of Hematological Disease, \\ and the Collaborative Innovation Center of Hematology, Soochow University, Suzhou, Jiangsu 215123; \\ ${ }^{3}$ Department of General Surgery, The First Affiliated Hospital of Soochow University, Suzhou, Jiangsu 215006, P.R. China
}

Received November 14, 2017; Accepted April 12, 2018

DOI: $10.3892 /$ or.2018.6374

\begin{abstract}
Vascular endothelial cadherin (VE-cadherin) was first found in vascular endothelial cells to maintain normal vascular structures and regulate endothelial cell permeability by homology adhesion. New evidence indicates that certain invasive tumor cells also express VE-cadherin, which is involved in vasculogenic mimicry to provide a blood supply required for tumor growth and metastasis. EC1 and EC3 domains of VE-cadherin were reported to be important for intercellular homology adhesion. In the present study, a monoclonal antibody specific to the outer-membrane immunoglobulin-like domains of VE-cadherin was generated and the binding epitope was identified as peptide LDREVVPWYNLTVEA in the EC4 domain. This antibody inhibited proliferation and capillary-like structure formation of lung cancer Glc-82 cells in 3D Matrigel culture in vitro. This effect was mediated by the inhibition of AKT phosphorylation. Our results suggested that the EC4 domain participates in VE-cadherin clustering and the antibody targeting the EC4 domain of VE-cadherin may be a promising anti-vasculogenic mimicry agent for cancer treatment.
\end{abstract}

Correspondence to: Dr Jianfeng Yang, Cyrus Tang Hematology Center and Ministry of Education Engineering Center of Hematological Disease, and the Collaborative Innovation Center of Hematology, Soochow University, 199 Renai Road, Suzhou, Jiangsu 215123, P.R. China

E-mail: yangjianfeng@suda.edu.cn

Dr Yang He, Ministry of Health Key Laboratory of Thrombosis and Hemostasis, Jiangsu Institute of Hematology, The First Affiliated Hospital of Soochow University, 188 Shizi Street, Suzhou, Jiangsu 215006, P.R. China

E-mail: heyang1963@163.com

${ }^{*}$ Contributed equally

Key words: epitope, lung cancer, monoclonal antibody, vasculogenic mimicry, VE-cadherin

\section{Introduction}

Vascular endothelial (VE)-cadherin is the main transmembrane protein sustaining intercellular adherens junctions in vascular endothelial cells where it plays a central role in modulating endothelial integrity and permeability (1). In vivo experimental models have demonstrated that mice deficient of VE-cadherin die in the embryo stage by severe vascular defects (2). Although VE-cadherin is considered specific for endothelial cells, its expression has also been found in several types of tumor cells such as melanoma, breast cancer and lung cancer (3-5). VE-cadherin is also expressed in a subset of acute lymphoblastic leukemia cells, where it contributes to cell survival (6). Surprisingly, VE-cadherin can be found in highly aggressive tumor cells but not in non-aggressive ones, which indicates that VE-cadherin may play various roles in tumor progression (7). In 1999, Maniotis et al presented a new concept, vasculogenic mimicry (VM), which describes the finding that tumor cells are able to self-assemble into tube-like structure independent of endothelial cells (7). Several endothelial-specific proteins were found in these VM tumor cells. Among them, VE-cadherin is considered most critical for VM as it was found that downregulation of VE-cadherin in melanoma led to the loss of VM formation (8). Thus, VE-cadherin has been used as a therapeutic target to control tumor angiogenesis in vivo (9).

VE-cadherins exhibit $\mathrm{Ca}^{2+}$-dependent homodimer interaction and cluster across cell membranes though their extracellular domains. Similar to N-cadherin and E-cadherin in the classic cadherin family, VE-cadherin also has five homologous domains in its extracellular region (named EC1 to EC5) (10). The cytoplasmic part of VE-cadherin contains domains interacting with $\beta$-catenin and plakoglobin $(11,12)$. There have been several studies that showed that the $\mathrm{N}$-terminal region corresponding to the $\mathrm{EC} 1$ domain of cadherins is responsible for intercellular hemophilic adhesion (13-16). Other research has found that there exists multiple binding sites in the ectodomain for cadherin adhesion. Antibodies directed to EC1 and EC3 affect VE-cadherin adhesion and clustering (17). However, whether other ECs are associated with VE-cadherin adhesion still needs to be elucidated. In the present study, we 
generated a monoclonal antibody binding to a novel epitope in the EC4 domain of VE-cadherin and demonstrated that the antibody inhibited the proliferation and vasculogenic mimicry of lung cancer Glc- 82 cells by modulating AKT phosphorylation. Our results suggest that the EC4 domain is involved in VE-cadherin adhesion and the monoclonal antibody may be promising for development for anti-vasculogenic mimicry in cancer treatment.

\section{Materials and methods}

Recombinant expression of the extracellular domain of $V E$-cadherin protein. The coding sequence for the whole extracellular region of VE-cadherin was amplified by reverse transcriptional PCR using total RNA extracted from human umbilical vein endothelial cells (HUVECs). The primer sequences were 5'-CCGGAATTCTGGATTTGGAACCAGA TGCAC-3' and 5'-CCGCTCGAGCAAGATGCTGTACTTG GTCAT-3' (EcoRI and XhoI sites are underlined). The PCR product was digested with restriction enzymes and ligated into the pET41a expression vector (Novagen, San Diego, CA, USA). The construct was transformed into DH5 $\alpha$ and the inserted fragment was verified by DNA sequencing. The recombinant protein was produced by transforming the expression construct into $E$. coli BL21 (DE3) followed by isopropyl $\beta$-D-1-thiogalactopyranoside (IPTG, $1 \mathrm{mM}$ ) induction. The bacteria were collected by centrifugation at 5,000 rpm for $10 \mathrm{~min}$ at $4^{\circ} \mathrm{C}$, and then sonicated in lysis buffer $(20 \mathrm{mM}$ $\mathrm{NaH}_{2} \mathrm{PO}_{4}, 10 \mathrm{mM}$ imidazole, $\mathrm{pH}$ 8.0). The supernatant which contained the VE-cadherin recombinant protein was collected by centrifugation at $13,000 \mathrm{rpm}$ for $20 \mathrm{~min}$ at $4^{\circ} \mathrm{C}$ and incubated with $\mathrm{Ni}^{2+}$-Sepharose beads for $3 \mathrm{~h}$ in an ice-water bath. The beads were washed with lysis buffer and the bound protein was eluted with elution buffer $\left(20 \mathrm{mM} \mathrm{NaH}_{2} \mathrm{PO}_{4}, 250 \mathrm{mM}\right.$ imidazole, $\mathrm{pH}$ 8.0). The solution with VE-cadherin fusion protein was dialyzed against $\mathrm{PBS}(\mathrm{pH} 7.4)$ overnight at $4^{\circ} \mathrm{C}$. The purified protein was analyzed by sodium dodecyl sulfatepolyacrylamide gel electrophoresis (SDS-PAGE) followed by Coomassie blue staining.

Production and characterization of monoclonal antibodies to the extracellular domain of VE-cadherin. BALB/c male mice were purchased from Shanghai SLAC Laboratory Animal company (Shanghai, China). The mice were housed in light-controlled room (lights on at 07:00, off at 19:00) at a room temperature of $23 \pm 1^{\circ} \mathrm{C}$ and a humidity of $50 \pm 0 \%$ with food and water available ad libitum. All animal procedures were conducted in strict accordance with the Animal Ethics Guidelines and approved by the Ethics Committee of Soochow University. Three BALB/c male mice (6 weeks old, $18-20 \mathrm{~g}$ ) were immunized with three injections of $100 \mu \mathrm{g}$ of the recombinant extracellular domain of VE-cadherin protein each time. Freund's complete adjuvant was used for the initial injection. The Freund's incomplete adjuvant was used for the second injection 28 days after the initial immunization. The first two immunizations were both administered subcutaneously. Four weeks later, $100 \mu \mathrm{g}$ of the recombinant protein was injected into the tail vein of the mice without adjuvant for the final boost. Three days later, mouse spleens were separated and splenocytes were isolated and fused with SP2/0 myeloma cells using PEG1500 (Sigma-Aldrich; Merck KGaA, Darmstadt, Germany). Hybridoma cells were selected in HAT medium (Thermo Fisher Scientific, Inc., Waltham, MA, USA). Hybridoma cells producing antibodies specific for recombinant VE-cadherin protein were identified by enzyme-linked immunosorbent assay (ELISA) and subcloned by two rounds limiting-dilution in 96-well plates.

Immunofluorescence. Cells $\left(5 \times 10^{3}\right)$ were inoculated in 96-well plates the day before and fixed with $4 \%$ paraformaldehyde at $4^{\circ} \mathrm{C}$ for $20 \mathrm{~min}$. Cells were washed with PBS three times and incubated with $2 \mathrm{C} 11,8 \mathrm{~A} 03$, or mouse $\operatorname{IgG}$ at $37^{\circ} \mathrm{C}$ for $1 \mathrm{~h}$. After washing, the cells were labeled with Alexa Fluor 488-conjugated goat anti-mouse $\operatorname{IgG}$ (1:500, Invitrogen; Thermo Fisher Scientific, Inc.). Nuclei were stained with DAPI (4',6-diamidino-2-phenylindole). The fluorescent images were captured using an inverted fluorescence microscope (DIML; Leica Microsystems, Wetzlar, Germany).

Flow cytometry. EA.hy926 cells $\left(3 \times 10^{6}\right)$ were trypsinized to suspension, washed with phosphate-buffered saline (PBS), and then incubated with $2 \mathrm{C} 11,8 \mathrm{~A} 03$, or mouse $\mathrm{IgG}(1 \mu \mathrm{g} / \mathrm{ml})$ at room temperature for $1 \mathrm{~h}$. Cells were washed with PBS and labeled with Alexa Fluor 488-conjugated goat anti-mouse IgG antibody for $1 \mathrm{~h}$ at room temperature. The reaction was stopped by addition of $500 \mu \mathrm{l}$ PBS and fluorescence was measured by flow cytometry (BD FACSCalibur; BD Biosciences, San Diego, CA, USA).

Preparation of recombinant human VE-cadherin fragments. Five pairs of primers were synthesized for amplification of the five extracellular domains (EC1-EC5) of VE-cadherin respectively (primer sequences are listed in Table I). The PCR products were constructed into the pET41a expression vector. The five EC domains were recombinantly expressed in BL21 (DE3) cells respectively under the induction of $1 \mathrm{mM}$ IPTG at $37^{\circ} \mathrm{C}$ for $3 \mathrm{~h}$. Bacterial cells were harvested and lysed in 1X Laemmli sample buffer (Bio-Rad Laboratories, Inc., Hercules, CA, USA) by boiling for $5 \mathrm{~min}$ and the soluble proteins were loaded on SDS-PAGE and the binding of $2 \mathrm{C} 11$ monoclonal antibody was assessed by western blot analysis.

\section{Epitope mapping of the 2 C11 monoclonal antibody}

Immunodot blot analysis. Seven consecutive 15-amino acid peptides spanning the whole EC4 domain were synthesized (Table II). Synthetic peptides (20, 40 and $80 \mathrm{ng})$ were spotted on a cellulose acetate membrane, the membrane was blocked with 5\% dried milk in PBS for $2 \mathrm{~h}$, and then incubated with 2C11 (1:500 dilution) for $1 \mathrm{~h}$ at room temperature followed by washing three times with PBS-T, and then incubated with HRP-conjugated goat anti-mouse IgG (1:10,000 dilution) for $1 \mathrm{~h}$. Enhanced chemiluminescence (ECL; Biological Industries, Kibbutz Beit Haemek, Israel) was conducted, and the signal was detected by Kodak Medical X-ray Processer (Kodak, Rochester, NY, USA) or Tanon 5200 AutoChemi Image system (Tanon Inc., Shanghai, China). The purified VE-cadherin fusion protein was used as the positive control.

Competitive ELISA. The synthetic peptides were preincubated with the $2 \mathrm{C} 11$ antibody for $1 \mathrm{~h}$, and then added to the 96-well 
Table I. Primer sequences for EC domain amplification.

cdh5-d1-f CCGGAATTCTGGATTTGGAACCAGATGCAC cdh5-d1-r CCGCTCGAGGAACACAGGCCAGTTGTCGTT cdh5-d2-f CCGGAATTCACGCATCGGTTGTTCAATGC cdh5-d3-r CCGCTCGAGGAAAATGGGGGGCTCGTC cdh5-d4-f CCGGAATTCCAGCAGCCTTTCTACCACTTCC cdh5-d4-r CCGCTCGAGCGGGGCATTGTCATTCTCAT cdh5-d5-f CCGGAATTCGAGTTTGCCAAGCCCTACCAG cdh5-d5-r CCGCTCGAGCAAGATGCTGTACTTGGTCAT

ELISA plates which were coated with the purified recombinant VE-cadherin protein. After reaction for $1 \mathrm{~h}$, the plate was washed three times with PBST and HRP-conjugated goat anti-mouse IgG (1:10,000 dilution) was added and incubated for $1 \mathrm{~h}$. After three times washing, TMB substrate was added into each wells to develop color, and the reaction was stopped by $2 \mathrm{~N} \mathrm{H}_{2} \mathrm{SO}_{4}$. The absorbance at $450 \mathrm{~nm}$ was measured using a spectrophotometer (Epoch; BioTek Instruments, Winooski, VT, USA).

Western blot analysis and RT-PCR detection of VE-cadherin expression. To prepare cell lysate, the cells were lysed with RIPA buffer (50 mM Tris-HCl, 1\% Triton X-100, 10\% glycerol, $150 \mathrm{mM} \mathrm{NaCl}$, proteinase inhibitor cocktail) for $30 \mathrm{~min}$ on ice and centrifuged at $13,000 \mathrm{rpm}$ for $10 \mathrm{~min}$ at $4^{\circ} \mathrm{C}$. The cell lysates containing a total protein amount of $25 \mu \mathrm{g}$ were placed onto SDS-PAGE gels for separation and transferred onto PVDF membranes by MiniProtean (Bio-Rad Laboratories). The membranes were blocked with 5\% non-fat milk in PBS for $2 \mathrm{~h}$, and then incubated with 2C11 (1:500 dilution) or 8A03 (1:200 dilution) for $1 \mathrm{~h}$ at room temperature followed by washing three times with PBS-T, and then incubated with HRP-conjugated goat anti-mouse IgG (1:10,000 dilution) for $1 \mathrm{~h}$. Proteins were detected by enhanced chemiluminescence (ECL; Biological Industries) by Kodak Medical X-ray Processer or Tanon 5200 AutoChemi Image system (Tanon Inc.).

RNA was isolated using TRIzol reagent. cDNA was obtained using RevertAid ${ }^{\mathrm{TM}}$ First Strand cDNA Synthesis kit (Fisher Scientific France, Illkirch-Graffenstaden, France). Semi-quantitative RT-PCR (30 cycles) was employed to analyze VE-cadherin gene expression using the gene-specific forward and reverse primers: 5'-CCGGAATTCTGGATTT GGAACCAGATGCAC-3' and 5'-CCGCTCGAGCAAGATG CTGTACTTGGTCAT-3'. The expression level of $\beta$-actin was used as the internal control.

$3 D$ cell culture. Tube-like formation in Matrigel was performed as previously described (18). In brief, Matrigel (150 $\mu \mathrm{l} / \mathrm{well}$ ) (BD Biosciences, Bedford, MA, USA) was added to 48-well plates on ice and allowed to polymerize for $30 \mathrm{~min}$ at $37^{\circ} \mathrm{C}$. Glc-82 cells and HUVECs were resuspended in DMEM containing MAb $2 \mathrm{C} 11,8 \mathrm{~A} 03$ or mouse IgG $(2 \mu \mathrm{g} / \mathrm{ml})$, which was plated onto the layer of Matrigel $\left(3 \times 10^{4}\right.$ cells/well). Matrigel cultures were incubated at $37^{\circ} \mathrm{C}$ and capillary tube formation was photographed at various time-points. Five visual fields (up, down, left, right and center) were randomly chosen from each well under an inverted microscope (Carl Zeiss, Oberkochen,
Table II. Synthetic peptide sequences used for epitope mapping.

\begin{tabular}{ll}
\hline Epitope & \multicolumn{1}{c}{ Amino acid sequence } \\
\hline D1 & QQPFYHFQLKENQKK \\
D2 & PLIGTVLAMDPDAAR \\
D3 & HSIGYSIRRTSDKGQ \\
D4 & FFRVTKKGDIYNEKE \\
D5 & LDREVVPWYNLTVEA \\
D6 & KELDSTGTPTGKESI \\
D7 & VQVHIEVLDENDNAP
\end{tabular}

Germany) to count the number of tube-like structures. The area covered by the tube network was quantified by ImageJ software (National Institutes of Health, Bethesda, MD, USA).

Cell proliferation assay. Cell proliferation was assessed using Cell Counting Kit-8 (CCK-8; Beyotime Institute of Biotechnology, Haimen, China) as previously described (19). Glc-82 cells were seeded in 96-well plates at a density of $5 \times 10^{3}$ cells per well. 2C11 antibody (at concentrations of 0.5 , 2 and $8 \mu \mathrm{g} / \mathrm{ml}$ ) was added into the cultured medium and incubated for 24,48 and $72 \mathrm{~h}$, respectively. CCK-8 solution $(10 \mu \mathrm{l})$ was added into each well and incubated for $3 \mathrm{~h}$ at $37^{\circ} \mathrm{C}$. The absorbance at $450 \mathrm{~nm}$ was measured with a spectrophotometer (Epoch; BioTek Instruments). Columns represented the mean percentage of optical density (OD) values relative to the negative control. All measurements were performed in triplicate and repeated at least three separate experiments.

Statistical analysis. Results are expressed as mean \pm SD (range) or percent. The difference between two groups was analyzed using the Student's paired t-test. A P-value $<0.05$ was considered statistically significant. All calculations were performed using GraphPad Prism software (GraphPad Software, Inc., San Diego, CA, USA).

\section{Results}

Expression and purification of recombinant VE-cadherin fusion protein. The cDNA encoding the extracellular domains (EC1 to EC5) of VE-cadherin was cloned into the pET41a vector. The histidine-tagged fusion protein was induced in BL21 (DE3) bacteria with $1 \mathrm{mM}$ IPTG. SDS-PAGE and Coomassie blue staining showed that the recombinant protein was successfully expressed at a molecular weight of approximately $70 \mathrm{kDa}$ and was mainly located in the precipitation fraction. The fusion protein in the precipitation fraction was purified by $\mathrm{Ni}^{2+}$-Sepharose beads (Fig. 1).

Specificity identification of monoclonal antibodies to $V E$-cadherin. The monoclonal antibody in the culture supernatants of hybridoma cells was detected by indirect ELISA. After two rounds of subcloning and detection, a stable positive hybridoma clone, designated as $2 \mathrm{C} 11$, was chosen to produce ascites. The monoclonal antibody in ascites was purified with protein $\mathrm{G}$ Sepharose and stored at a final concentration of $0.35 \mathrm{mg} / \mathrm{ml}$. 


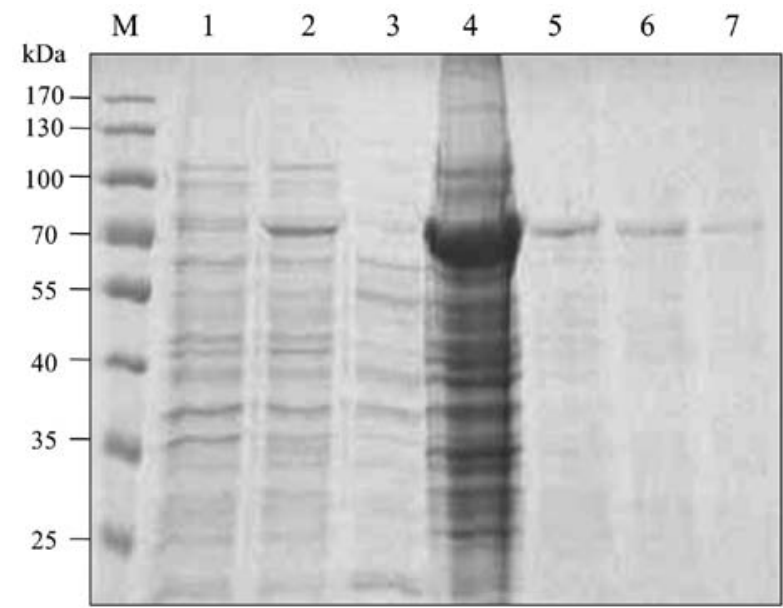

Figure 1. SDS-PAGE analysis of the VE-cadherin protein fragment expressed in BL21 (DE3). M, protein marker; lane 1, total lysate without IPTG induction; lane 2, total lysate after $1 \mathrm{mM}$ IPTG induction; lane 3, soluble supernatant; lane 4, insoluble fraction after $1 \mathrm{mM}$ IPTG induction; lanes 5-7, purified recombinant protein. VE-cadherin, vascular endothelial cadherin.

To analyze whether 2C11 could bind native VE-cadherin, EA.hy926 cells, HUVECs and HepG2 cell lysates were prepared for western blot analysis. The results showed that 2C11 specifically recognized a protein with a molecular weight of approximately $130 \mathrm{kDa}$ in EA.hy926 and HUVEC cells, but not in HepG2 cells. Commercial antibody 8A03 was used as a positive control (Fig. 2A).
To further explore the binding specificity of 2C11 antibody to VE-cadherin, immunofluorescence and flow cytometric analysis were performed. Flow cytometry demonstrated that 2C11 could recognize VE-cadherin located on the endothelial cell surface (Fig. 2B). Results of the immunofluorescence showed positive green fluorescence on the EA.hy926 cell surface labeled with $2 \mathrm{C} 11$, while no fluorescence was detected on cells labeled with mouse IgG (Fig. 2C). These results suggest that the $2 \mathrm{C} 11$ antibody recognized the VE-cadherin native protein.

Epitope mapping with synthetic peptides. To explore the binding epitope of the $2 \mathrm{C} 11$ antibody, five fragments representing a different region of VE-cadherin extracellular domain (EC1-EC5) were recombinantly expressed in E. coli. The predicted molecular weights of the resulting recombinant proteins were $45,45,50,45$ and $45 \mathrm{kDa}$, respectively (Fig. 3A). Western blot analysis showed that mAb 2C11 specifically recognized the EC4 fragment (Fig. 3B) which contains 105 amino acids. Next, seven consecutive peptides, each comprised of 15 amino acids, were synthesized (sequences are listed in Table I). Dot blot and competitive ELISA showed that 2 C11 specifically recognized the fifth peptide D5. These results demonstrated that the D5 peptide LDREVVPWYNLTVEA was the binding epitope of the 2C11 antibody (Fig. 3C and D).

2 C11 inhibits vasculogenic mimicry formation of Glc-82 cells in vitro. Vasculogenic mimicry was observed in some aggressive tumor cells. Surface expression of VE-cadherin
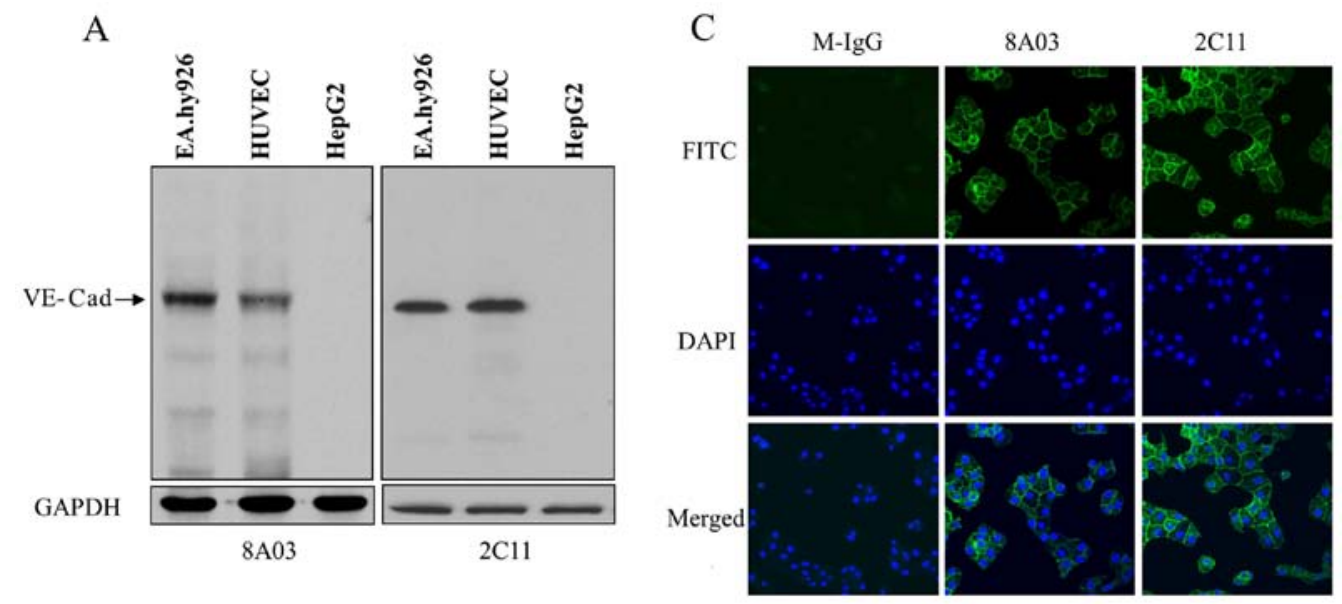

B
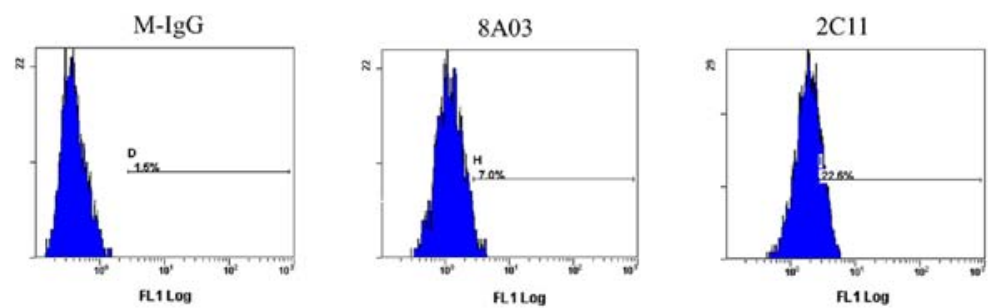

Figure 2. 2C11 recognizes the VE-cadherin protein as determined by western blot analysis, flow cytometry and immunofluorescence analysis. (A) 2C11 recognized a protein with a molecular weight of $\sim 130 \mathrm{kDa}$ in EA.hy926 cells, HUVECs, but not in HepG2 cells. (B) Flow cytometric analysis. EA.hy926 cells were labeled with the $2 \mathrm{C} 11$ antibody. Mouse $\mathrm{IgG}$ was used as the negative control. (C) Immunofluorescence analysis of the $2 \mathrm{C} 11$ antibody recognized the VE-cadherin protein in EA.hy926 cells. The commercial anti-VE-cadherin antibody 8A03 was used as a positive control in the experiments above. Data are from three representative experiments. VE-cadherin, vascular endothelial cadherin; HUVECs, human umbilical vein endothelial cells. 
A

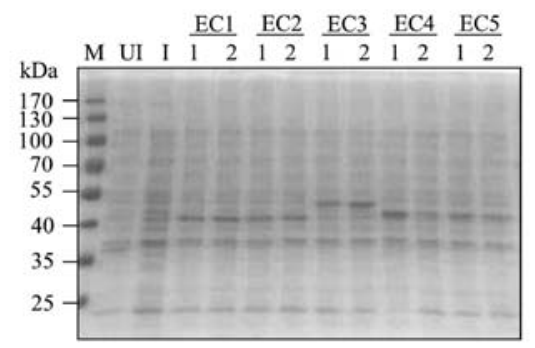

$\mathrm{C}$

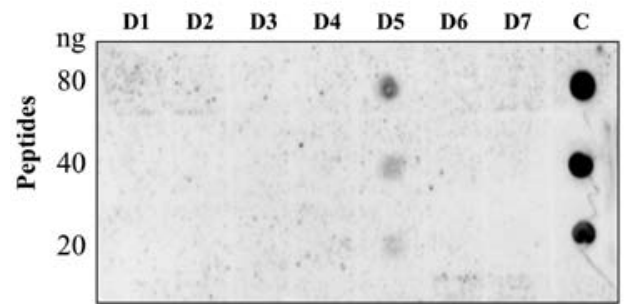

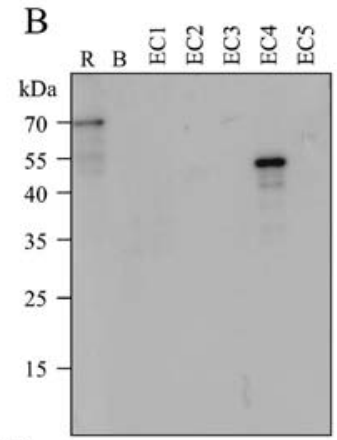

$\mathrm{D}$

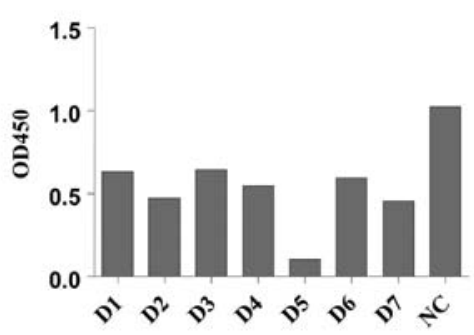

Figure 3. Epitope mapping of the 2C11 antibody. (A) The five extracellular domain (EC1 to EC5) fragments were expressed in E. coli, respectively. Two expression clones of each EC domain were induced: 'UI' represents the uninduced empty vector, 'I' represents the induced empty vector. (B) Western blot analysis showed that mAb 2C11 specifically recognized the EC4 protein fragment. (C) Synthetic peptides (D1-D7) were dot blotted to cellulose acetate membrane. Result shows that $2 \mathrm{C} 11$ specifically recognized the D5 peptide in a concentration-dependent manner. The whole extracellular region fragment was used as a positive control. (D) Competition ELISA displayed that the D5 peptide competitively inhibited the binding of 2C11 to the VE-cadherin protein fragment. Data are from three representative experiments. VE-cadherin, vascular endothelial cadherin; C, control; NC, negative control.
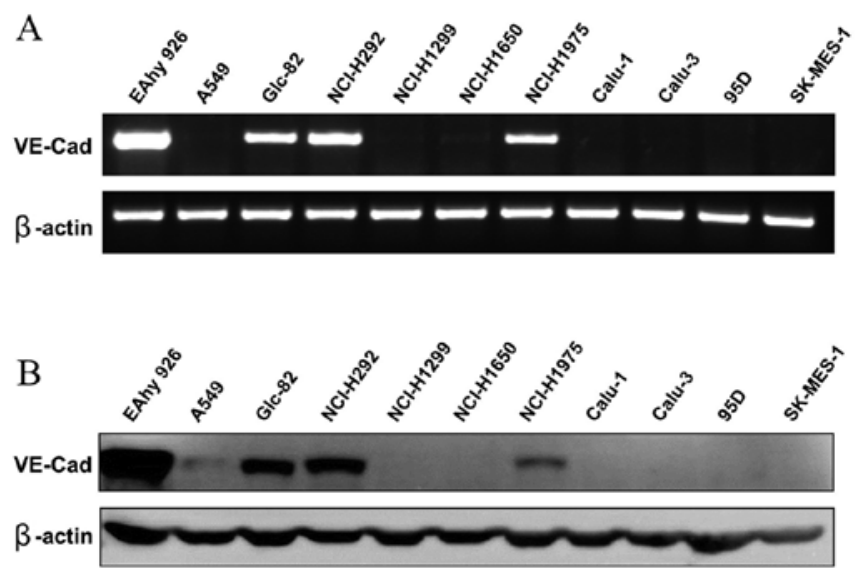

Figure 4. Identification of VE-cadherin expression in different cell lines. (A) RT-PCR showed that VE-cadherin mRNA was expressed in 3 out of the10 lung cancer cell lines tested. (B) Western blot analysis showed that $2 \mathrm{C} 11$ recognized a protein with a molecular weight of $\sim 130 \mathrm{kDa}$ in GLc- 82 , NCI-H292 and NCI-H1975 cells, but not in the other 7 lung cancer cell lines tested. EA.hy926 endothelial cells were used as a positive control. VE-cadherin, vascular endothelial cadherin.

on tumor cells is considered most important for VM formation. Whether the 2C11 monoclonal antibody has biological activity on VM of tumor cells is unknown. Here we examined several poorly differentiated lung adenocarcinoma cell lines; among them, Glc-82 cells were found to express VE-cadherin by RT-PCR and western blot analysis (Fig. 4A and B). Next, we tested the effect of $2 \mathrm{C} 11$ on tube-like structure formation of Glc-82 lung cancer cells. The results showed that $2 \mathrm{C} 11$, but not mouse $\mathrm{IgG}$ and $8 \mathrm{~A} 03$ antibody, significantly prevented tubular network formation and reduced the size and length
A

है

$\mathrm{mIgG}$
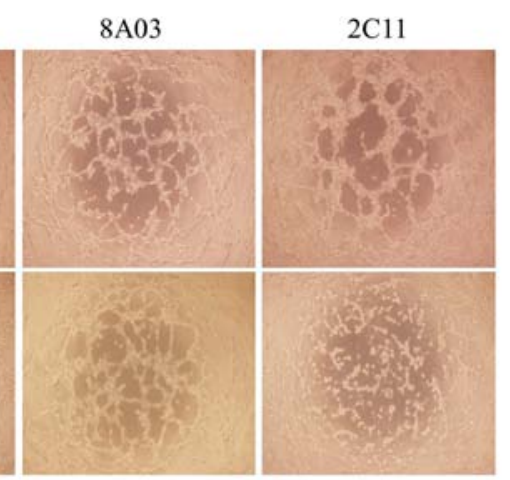

B

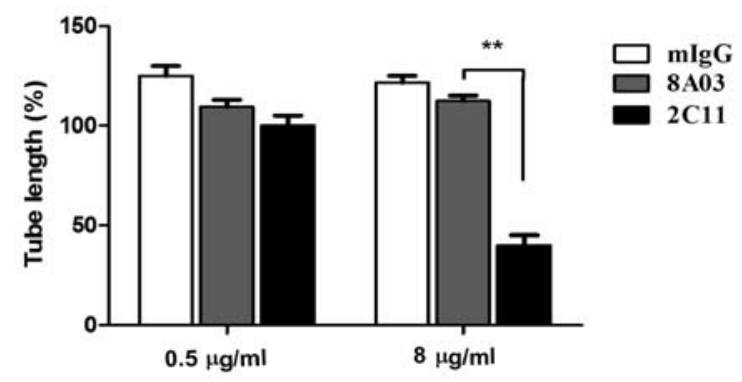

Figure 5. VE-cadherin antibody 2C11 inhibits VM structure formation of Glc-82 lung cancer cells. Tubular network formation on Matrigel was (A) photographed and (B) quantified at $24 \mathrm{~h}$. A commercial antibody 8A03 was used as a positive control and mouse $\mathrm{IgG}$ as a negative control. All data are presented as the mean \pm SE from three different experiments in duplicate. ${ }^{* *} \mathrm{P}<0.01$. VE-cadherin, vascular endothelial cadherin; VM, vascular mimicry.

of the cords of Glc-82 cells (Fig. 5A and B). These results demonstrated that the anti-VE-cadherin mAb 2C11 inhibited VM formation of lung cancer cells and may be promising to 
A

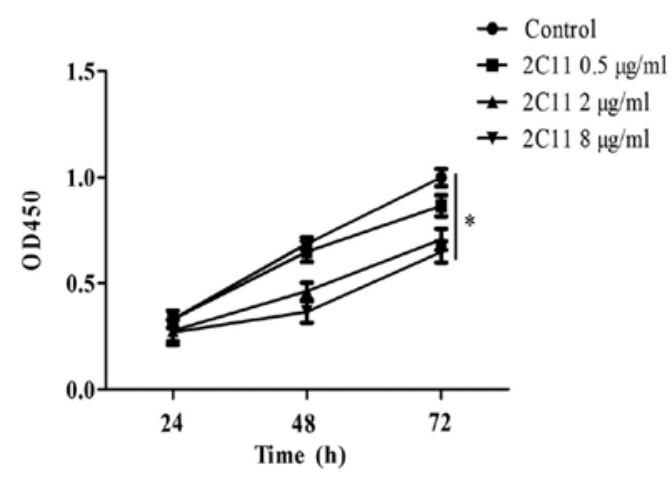

B

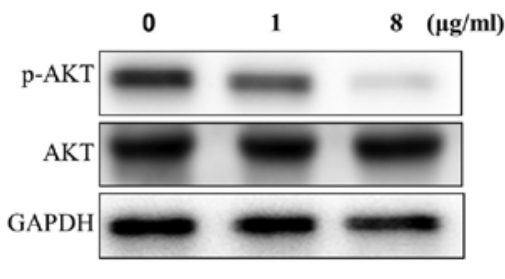

$\mathrm{C}$

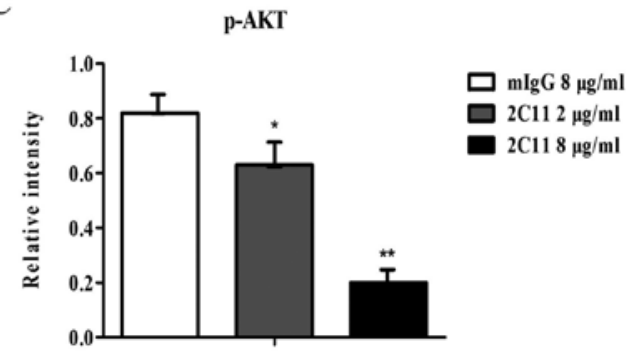

Figure 6. Effects of $2 \mathrm{C} 11$ on cell proliferation of Glc-82 lung cancer cells. (A) Glc-82 cells were cultured with the $2 \mathrm{C} 11$ antibody $(0.5,2$ and $8 \mu \mathrm{g} / \mathrm{ml})$ for 24 , 48 and $72 \mathrm{~h}$. Cell proliferation was measured by CCK-8 assay. Mouse IgG was used as the negative control. (B and C) Cells were treated for $24 \mathrm{~h}$ with IgG control, 2C11 (2 and $8 \mu \mathrm{g} / \mathrm{ml}$ ). Cell lysates were blotted with a p-AKT antibody, using GAPDH as a loading control. A representative blot is shown with the mean densitometric analysis of all experiments. Data are represented as mean $\pm \mathrm{SE}$ from three independent experiments. ${ }^{*} \mathrm{P}<0.05 ;{ }^{* *} \mathrm{P}<0.01$.

be developed as a therapeutic antibody for aggressive lung cancer.

Effect of 2 C11 on Glc-82 cell proliferation. To examine the effect of $2 \mathrm{C} 11$ on cell proliferation, a proliferation assay was performed using CCK-8 reagent. The $2 \mathrm{C} 11$ antibody was added into the Glc- 82 cell culture medium and incubated for different time periods. Mouse $\operatorname{IgG}(10 \mu \mathrm{g} / \mathrm{ml})$ was used as the negative control. Data showed that the proliferation rates of Glc-82 cells were slower after treatment of the $2 \mathrm{C} 11$ antibody compared to the negative control (100\% proliferation, Fig. 6A). The result indicated that the $2 \mathrm{C} 11$ antibody inhibited the proliferation of Glc-82 cells.

$m A b 2 C 11$ inhibits the phosphorylation of AKT. The $2 \mathrm{C} 11$ antibody inhibits Glc-82 cell proliferation, while the molecular mechanism is unknown. PI3K/AKT signaling is the classical pathway involved in cell proliferation. In the present study, we examined the effect of $2 \mathrm{C} 11$ on the AKT signaling pathway. We found that following addition of different concentrations of the 2C11 antibody, the phosphorylation level of AKT was decreased (Fig. 6B and $\mathrm{C}$ ). This result demonstrated that the $2 \mathrm{C} 11$ antibody inhibited Glc-82 cell proliferation via reducing AKT phosphorylation.

\section{Discussion}

Cadherins are a family of calcium-dependent, cell-cell adhesion molecules, which are located at adherens junctions that mediate adhesive intercellular interactions and provide a dynamic cellcell contact and positional clues to cells during development. VE-cadherin, a unique member of the cadherin family, is expressed specifically on endothelial cells during the development of embryo vasculature which has been shown to play a central role in controlling endothelial monolayer integrity, junction permeability, cell growth and angiogenesis. However, the molecular mechanism of VE-cadherin homophilic binding has not yet been fully elucidated. Previous research has shown that two monoclonal antibodies BV6 and Cad 5 to VE-cadherin could increase paracellular permeability and inhibit VE-cadherin reorganization. Epitope mapping studies defined the sequences TIDLRY located on EC3 and KVFRVDAETGDVFAI on EC1 as the binding domain of BV6 and Cad 5, respectively (17), indicating that $\mathrm{EC} 1$ and $\mathrm{EC} 3$ extracellular regions are important for VE-cadherin intercellular homophilic binding. However, whether other extracellular domains are involved in the interaction is not yet clear. In the present study, we prepared a monoclonal antibody (2C11) to VE-cadherin and mapped its binding epitope to LDREVVPWYNLTVEA in the EC4 domain of VE-cadherin, and functional assays revealed that this antibody was able to block VE-cadherin-mediated cell adhesion, suggesting that besides EC1 and EC3, EC4 domain is also critical for the molecular function of VE-cadherin.

Vasculogenic mimicry is found in certain aggressive tumors, in which VE-cadherin plays a key role. The effect of $2 \mathrm{C} 11$ antibody on VM is not known. In this study, we examined the VE-cadherin expression in several lung cancer cell lines both at the mRNA and protein levels and found that VE-cadherin was expressed in Glc-82 cells. Moreover, our data demonstrated that the 2C11 antibody inhibited VM-like structure of Glc-82 cells on 3D Matrigel. Mechanistically, 2C11 may inhibit cellcell adhesion necessary for VM by blockage of intercellular VE-cadherin interaction. Furthermore, 2C11 may also affect Glc-82 cell biological functions. It has been reported that knockdown of VE-cadherin inhibited the proliferation and promoted the apoptosis of esophageal squamous cell carcinoma cells (ESCCs) and osteosarcoma cells $(20,21)$. Here we examined the effect of the $2 \mathrm{C} 11$ antibody on cell proliferation using CCK-8 assay. Our result showed that the 2C11 antibody inhibited Glc-82 cell proliferation.

PI3K/AKT signaling is the most classic pathway involved in cell proliferation. In vascular endothelial cells, VE-cadherin interacts with VEGFR2 and inhibits VEGFR2 activation and downstream AKT phosphorylation (22). In contrast, Taddei et al 
reported that VE-cadherin clustering induced claudin-5 expression in endothelial cells by activation of PI3K/AKT signaling, and AKT phosphorylation was reduced when VE-cadherin was knocked down (23). Our result showed that the 2C11 antibody inhibited phosphorylation of AKT in Glc-82 cells, which may account for the mechanism of the growth inhibitory effect of the $2 \mathrm{C} 11$ antibody on Glc-82 lung cancer.

In conclusion, we generated a novel monoclonal antibody to VE-cadherin and mapped its binding epitope to the fourth extracellular domain (EC4). We found that this antibody inhibited vasculogenic mimicry of aggressive lung cancer Glc-82 cells, suggesting that the antibody blocked VE-cadherin intercellular clustering. This is the first report to show that the EC4 domain is involved in VE-cadherin clustering. Furthermore, we found that Glc-82 cell growth and AKT phosphorylation were inhibited by the $2 \mathrm{C} 11$ antibody. These results may provide a candidate approach to control VE-cadherin-positive aggressive tumors.

\section{Acknowledgements}

Not applicable.

\section{Funding}

The present study was supported in part by grants from the Priority Academic Program Development of Jiangsu Higher Education Institutions (PAPD), the National Natural Science Foundation of China (nos. 81773356, 81673096, 81600565, 81700129 and 81700235), the Natural Science Foundation (nos. BK20171204 and BK20150296), the Science and Technology Department (no. BY2015039-C03) of Jiangsu and the Science and Technology Department of Suzhou (no. SYS201704).

\section{Availability of data and materials}

Available from the corresponding author upon reasonable request.

\section{Authors' contributions}

Acquisition, analysis and interpretation of data and the drafting of the article were carried out by JD and XJ. Acquisition of data was undertaken by BZ and JH. Design of the experiments, acquisition, analysis and interpretation of data, drafting and revising the article were conducted by JY and YH. All authors read and approved the manuscript and agree to be accountable for all aspects of the research in ensuring that the accuracy or integrity of any part of the work are appropriately investigated and resolved.

\section{Ethics approval and consent to participate}

Approval has been obtained by the Ethics Committees of Soochow University, Suzhou.

\section{Consent for publication}

Not applicable.

\section{Competing interests}

The authors declare that they have no conflict of interests.

\section{References}

1. Dejana E: Endothelial adherens junctions: Implications in the control of vascular permeability and angiogenesis. J Clin Invest 98: 1949-1953, 1996.

2. Carmeliet P, Lampugnani MG, Moons L, Breviario F, Compernolle V, Bono F, Balconi G, Spagnuolo R, Oosthuyse B, Dewerchin M, et al: Targeted deficiency or cytosolic truncation of the VE-cadherin gene in mice impairs VEGF-mediated endothelial survival and angiogenesis. Cell 98: 147-157, 1999.

3. Hendrix MJ, Seftor EA, Hess AR and Seftor RE: Vasculogenic mimicry and tumour-cell plasticity: Lessons from melanoma. Nat Rev Cancer 3: 411-421, 2003.

4. Rong X, Huang B, Qiu S, Li X, He L and Peng Y: Tumorassociated macrophages induce vasculogenic mimicry of glioblastoma multiforme through cyclooxygenase-2 activation. Oncotarget 7: 83976-83986, 2016.

5. Williamson SC, Metcalf RL, Trapani F, Mohan S, Antonello J, Abbott B, Leong HS, Chester CP, Simms N, Polanski R, et al: Vasculogenic mimicry in small cell lung cancer. Nat Commun 7: 13322, 2016.

6. Wang L, O'Leary H, Fortney $\mathrm{J}$ and Gibson LF: $\mathrm{Ph}^{+} / \mathrm{VE}$-cadherin ${ }^{+}$ identifies a stem cell like population of acute lymphoblastic leukemia sustained by bone marrow niche cells. Blood 110: 3334-3344, 2007.

7. Maniotis AJ, Folberg R, Hess A, Seftor EA, Gardner LM, Pe'er J, Trent JM, Meltzer PS and Hendrix MJ: Vascular channel formation by human melanoma cells in vivo and in vitro: Vasculogenic mimicry. Am J Pathol 155: 739-752, 1999.

8. Delgado-Bellido D, Serrano-Saenz S, Fernández-Cortés M and Oliver FJ: Vasculogenic mimicry signaling revisited: Focus on non-vascular VE-cadherin. Mol Cancer 16: 65, 2017.

9. Corada M, Zanetta L, Orsenigo F, Breviario F, Lampugnani MG, Bernasconi S, Liao F, Hicklin DJ, Bohlen P and Dejana E: A monoclonal antibody to vascular endothelial-cadherin inhibits tumor angiogenesis without side effects on endothelial permeability. Blood 100: 905-911, 2002.

10. Yagi T and Takeichi M: Cadherin superfamily genes: Functions, genomic organization, and neurologic diversity. Genes Dev 14 1169-1180, 2000.

11. Garrett JP, Lowery AM, Adam AP, Kowalczyk AP and Vincent PA: Regulation of endothelial barrier function by p120catenin·VE-cadherin interaction. Mol Biol Cell 28: 85-97, 2017.

12. Venkiteswaran K, Xiao K, Summers S, Calkins CC, Vincent PA, Pumiglia K and Kowalczyk AP: Regulation of endothelial barrier function and growth by VE-cadherin, plakoglobin, and beta-catenin. Am J Physiol Cell Physiol 283: C811-C821, 2002.

13. Renaud-Young $M$ and Gallin WJ: In the first extracellular domain of E-cadherin, heterophilic interactions, but not the conserved His-Ala-Val motif, are required for adhesion. J Biol Chem 277: 39609-39616, 2002.

14. Patel SD, Ciatto C, Chen CP, Bahna F, Rajebhosale M, Arkus N, Schieren I, Jessell TM, Honig B, Price SR, et al: Type II cadherin ectodomain structures: Implications for classical cadherin specificity. Cell 124: 1255-1268, 2006.

15. Harrison OJ, Bahna F, Katsamba PS, Jin X, Brasch J, Vendome J, Ahlsen G, Carroll KJ, Price SR, Honig B, et al: Two-step adhesive binding by classical cadherins. Nat Struct Mol Biol 17: 348-357, 2010.

16. Dalle Vedove A, Lucarelli AP, Nardone V, Matino A and Parisini E: The X-ray structure of human P-cadherin EC1-EC2 in a closed conformation provides insight into the type I cadherin dimerization pathway. Acta Crystallogr F Struct Biol Commun 71: 371-380, 2015.

17. Corada M, Liao F, Lindgren M, Lampugnani MG, Breviario F, Frank R, Muller WA, Hicklin DJ, Bohlen P and Dejana E: Monoclonal antibodies directed to different regions of vascular endothelial cadherin extracellular domain affect adhesion and clustering of the protein and modulate endothelial permeability. Blood 97: 1679-1684, 2001.

18. Collet G, Szade K, Nowak W, Klimkiewicz K, El Hafny-Rahbi B, Szczepanek K, Sugiyama D, Weglarczyk K, Foucault-Collet A, Guichard A, et al: Endothelial precursor cell-based therapy to target the pathologic angiogenesis and compensate tumor hypoxia. Cancer Lett 370: 345-357, 2016. 
19. Zhang J, Wang J,Jiang JY,Liu SD, Fu K and Liu HY: Tanshinone IIA induces cytochrome $c$-mediated caspase cascade apoptosis in A549 human lung cancer cells via the JNK pathway. Int J Oncol 45: 683-690, 2014.

20. Tang NN, Zhu H, Zhang HJ, Zhang WF, Jin HL, Wang L, Wang P, He GJ, Hao B and Shi RH: HIF-1 $\alpha$ induces VE-cadherin expression and modulates vasculogenic mimicry in esophageal carcinoma cells. World J Gastroenterol 20: 17894-17904, 2014.

21. Zhang LZ, Mei J, Qian ZK, Cai XS, Jiang Y and Huang WD: The role of VE-cadherin in osteosarcoma cells. Pathol Oncol Res 16 $111-117,2010$.
22. Tian Y, Gawlak G, O'Donnell JJ III, Birukova AA and Birukov KG: Activation of vascular endothelial growth factor (VEGF) receptor 2 mediates endothelial permeability caused by cyclic stretch. J Biol Chem 291: 10032-10045, 2016.

23. Taddei A, Giampietro C, Conti A, Orsenigo F, Breviario F, Pirazzoli V, Potente M, Daly C, Dimmeler S and Dejana E: Endothelial adherens junctions control tight junctions by VE-cadherin-mediated upregulation of claudin-5. Nat Cell Biol 10: 923-934, 2008. 